\title{
Topological states in engineered atomic lattices
}

\author{
Robert Drost, Teemu Ojanen, Ari Harju` and Peter Liljeroth ${ }^{\star}$
}

\begin{abstract}
Topological materials exhibit protected edge modes that have been proposed for applications in, for example, spintronics and quantum computation. Although a number of such systems exist $^{1-6}$, it would be desirable to be able to test theoretical proposals in an artificial system that allows precise control over the key parameters of the model ${ }^{7}$. The essential physics of several topological systems can be captured by tight-binding models, which can also be implemented in artificial lattices ${ }^{6,7}$. Here, we show that this method can be realized in a vacancy lattice in a chlorine monolayer on a $\mathrm{Cu}(100)$ surface. We use low-temperature scanning tunnelling microscopy (STM) to fabricate such lattices with atomic precision and probe the resulting local density of states (LDOS) with scanning tunnelling spectroscopy (STS). We create analogues of two tight-binding models of fundamental importance: the polyacetylene (dimer) chain with topological domain-wall states, and the Lieb lattice with a flat electron band. These results provide an important step forward in the ongoing effort to realize designer quantum materials with tailored properties.
\end{abstract}

The topological theory of matter has its roots in the 70 s and 80 s studies of polyacetylene ${ }^{8}$, the quantum Hall effect ${ }^{9}$ and superfluid ${ }^{3} \mathrm{He}$ (ref. 10). The interest in the field exploded after the 2006 discovery of topological insulators ${ }^{2,3}$. Crystalline solids exhibit a spectrum of energy bands constrained by the material's symmetries. Topological properties of the band structure give rise to protected boundary states that are the hallmark of topological materials. Well-known examples include chiral and helical states in quantum (spin) Hall systems and Majorana modes in topological superconductors ${ }^{1,9,11-14}$. A central goal of research has become to identify systems with a topological spectrum.

Theories of topological materials ${ }^{15}$ are often formulated using tight-binding models describing hopping between localized electronic orbitals. This means that, given sufficient control, one can implement these models by assembling the corresponding structure from individual constituents that are suitably coupled. It is hence possible to build 'designer quantum materials' based on specific Hamiltonians through atomic assemblies. Although there has been considerable success in this direction using ultracold atomic gases, STM can be used to fabricate and characterize structures at the atomic scale in the solid state ${ }^{7,16-19}$. Recently, it was shown that vacancy defects in the $\mathrm{c}(2 \times 2)$ chlorine superstructure on $\mathrm{Cu}(100)$ make an excellent system for large-scale atomic assembly ${ }^{20}$. We show that individual $\mathrm{Cl}$ vacancies host a well-defined vacancy state below the band edge of the chlorine layer which interact when sufficiently close to each other. We demonstrate that it is possible to construct coupled lattices and implement two Hamiltonians of general interest, the Su-Schrieffer-Heeger (SSH) dimer chain and the two-dimensional Lieb lattice.

An overview image of the chlorine layer with several vacancies and typical $\mathrm{d} I / \mathrm{d} V$ spectra of the vacancy states are shown in Fig. 1a (see Supplementary Information for additional spectroscopy). The surface is characterized by a slowly varying density of states around the Fermi energy and a prominent band edge at about 3.5 V (green line in Fig. 1a). The vacancy sites host an electronic state split off from this band edge (purple line).

We constructed vacancy dimers at different separations through lateral manipulation and investigated their properties. Figure $1 \mathrm{c}-\mathrm{f}$ shows conductance spectra and maps acquired on two vacancy dimers separated by one or two chlorine sites. Coupling between the vacancies modifies the spectra considerably. Instead of a single resonance, we now find one component above and one component below $3.5 \mathrm{~V}$. Conductance maps acquired at the respective energies, presented in Figs $1 \mathrm{~d}$ and 1f, clearly show that the lower-energy resonance is found in between the vacancy sites, while the higher-energy component is stronger on the outer edges (the high-energy component of the short dimer lies within the conduction band of the chlorine layer and thus gives poor contrast). These are clear signatures of the formation of bonding and anti-bonding combinations of the vacancy state wavefunctions $^{21,22}$. On the basis of our data, we estimate the following hopping amplitudes: $t_{\text {short }} \approx 0.14 \mathrm{eV}, t_{\text {long }} \approx 0.04 \mathrm{eV}$ and $t_{\text {long }} / t_{\text {short }} \approx 0.28$ (see Supplementary Information for details). These results establish our premise that the vacancy states may be used to construct interacting lattices.

One of the simplest models in which topological states emerge is the SSH dimer chain, originally developed to describe the polyacetylene molecule and the soliton states within it $^{8}$. The topologically protected domain-wall states in the SSH model provide a condensed-matter realization of the domain-wall states in the Jackiw-Rebbi model of the $(1+1)$ dimensional Dirac equation with a mass kink ${ }^{23}$. The SSH Hamiltonian is given by:

$$
\mathscr{H}=-\sum t_{i} \hat{c}_{i}^{\dagger} \hat{c}_{i+1}+\text { h.c. }
$$

where the hopping parameter alternates between $t_{i}=t_{0} \pm \delta t$ for even/odd $i$, and $c_{i}^{\dagger}$ and $c_{i}$ are electronic creation and annihilation operators, respectively, at site $i$. The system has a bandgap determined by $|\delta t|$. The chain exists in two phases depending on the sign of $\delta t$ and distinguished by a topological index, the winding number. Physically, this means that the two phases are distinguished by the location of the strong bonds. Mid-gap states are expected on the boundary between these two phases where $\delta t$ changes sign. The characteristic localization length of the domain-wall states in units of lattice constant is $\xi=\left|t_{0}-\delta t\right| /|\delta t|$. In our experiment, we expect strongly localized states as $\delta t \approx 0.55 t_{0}$.

The structure and a set of spectra for a short dimer chain can be found in Fig. 2a,b. Two sets of states can be seen to extend throughout the chain above and below the vacancy state energy. In analogy to the SSH model, we refer to the region in between these two bands as the gap and to states within it as sub-gap states 
a
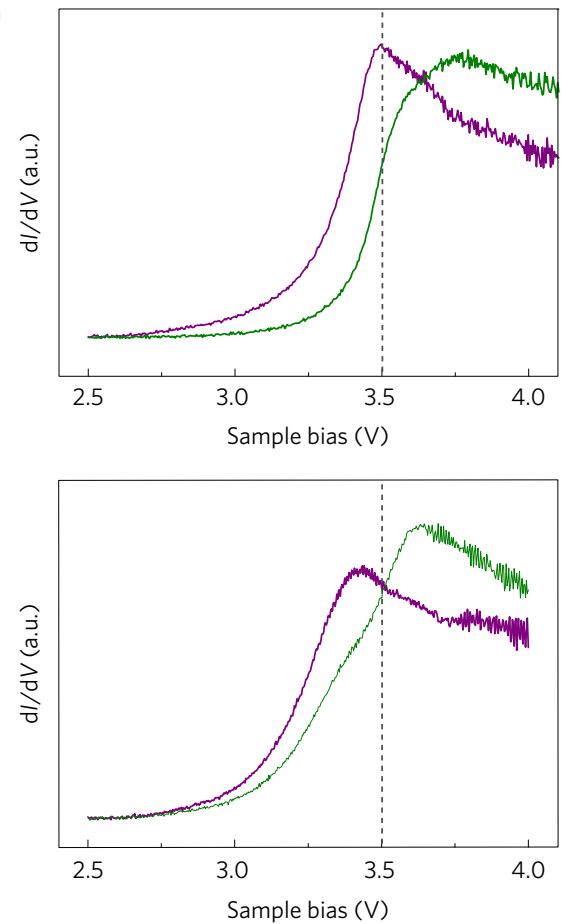

e

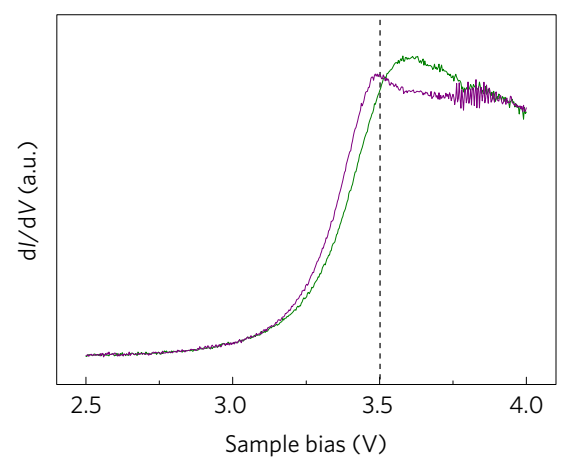

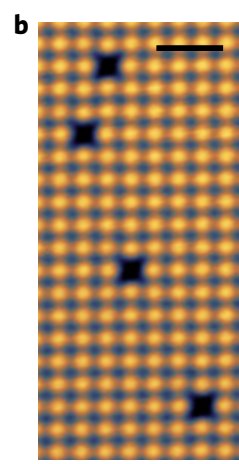

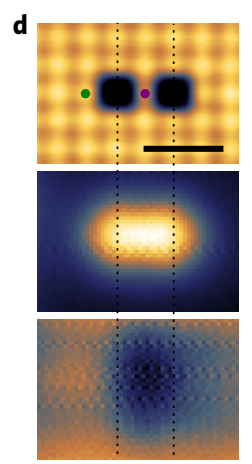

f

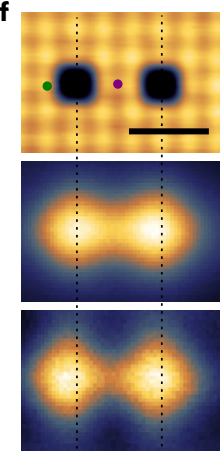

Figure 1 | Electronic structure of vacancies and vacancy dimers. a, Typical conductance spectra acquired on the chlorine layer (green) and on an isolated vacancy (purple). b, Overview image of the chlorine-terminated $\mathrm{Cu}(100)$ surface with a few vacancies visible. c, Conductance spectra acquired at the locations marked in $\mathbf{d}$. $\mathbf{d}$, Top: vacancy dimer separated by a single $\mathrm{Cl}$ site. The coloured dots mark the locations of the point spectra in panel c. Centre: conductance map at $3.3 \mathrm{~V}$ showing the bonding states. Bottom: conductance map at $3.6 \mathrm{~V}$ showing the anti-bonding state. Note that this state lies within the conduction band for this structure and hence provides poor contrast ${ }^{22}$. e, Conductance spectra acquired at the locations marked in f. f. Top: vacancy dimer separated by two $\mathrm{Cl}$ sites. The coloured dots mark the locations of the point spectra in $\mathbf{e}$. Centre: conductance map at $3.45 \mathrm{~V}$ showing the bonding states. Bottom: conductance map at $3.53 \mathrm{~V}$ showing the anti-bonding state. The dashed line marks the energy of the single vacancy state. All scale bars, $1 \mathrm{~nm}$.

despite their location far above the Fermi energy. According to our estimation of the coupling constants, we expect a bandgap of

$$
\Delta E_{\text {min }}=2\left(t_{\text {short }}-t_{\text {long }}\right) \approx 0.2 \mathrm{eV}
$$

which agrees with our experimental findings. No sub-gap states exist within this structure.

We constructed a chain containing two domain walls (see Fig. 2c). The lower panel of Fig. $2 c$ shows a constant height conductance map of this structure acquired at $3.53 \mathrm{~V}$. The two domain walls are clearly visible at this bias. We confirmed the

existence of mid-gap modes through point spectroscopy. Figure $2 \mathrm{~d}$ shows a stacked contour plot of conductance spectra acquired along the chain. The bulk of the chain shows the same signatures as that shown in Fig. 2b. At the location of the domain walls, however, a single resonance centred around the mid-gap energy emerges. These are the topologically protected mid-gap states predicted by the SSH model. To show that the presence of topological states is independent of the chain form, we also studied a ring-like structure with two embedded phase boundaries. This structure can be found in Fig. 2e with the corresponding conductance map at the mid-gap energy. As expected, mid-gap states located on the domain-wall sites are found.

To show that the concept of lattice engineering can be generalized to more complex systems, we constructed a Lieb lattice structure from chlorine vacancies. This is a line-centred square structure with a three-atom unit cell, resulting in a fermionic system with a lattice pseudospin of 1 . The band structure in the infinite limit consists of a Dirac cone on the corners of the first Brillouin zone intersected by a flat band at the Dirac energy ${ }^{24}$. Such flat bands are prone to electronic instabilities near half-filling and have been suggested to yield magnetic or superconducting order ${ }^{25-28}$. The presence of flat bands could also enhance the properties of superconducting materials by increasing the critical temperature ${ }^{29,30}$. Depending on the nature of the interactions in the lattice, topological states may also emerge $e^{26,31}$. Although Lieb lattices have been studied in optical lattices and ultracold atomic gases ${ }^{32,33}$, no realization using electronic states exist to our knowledge.

A small Lieb lattice can be seen in Fig. 3a with point spectra taken on the A, B and C sites of the three-atom unit cell presented in panel $b$. Figure 3 shows constant height conductance maps acquired at different bias voltages. At $2.85 \mathrm{~V}$ and $3.15 \mathrm{~V}$, the maps reveal an extended electronic state with higher intensity on the A sites. This finding is reproduced well by our tight-binding simulation (see below). The signal distribution we observe here is characteristic of the dispersive bands in the Lieb lattice which converge to form the Dirac cone. The map taken at a bias of $3.5 \mathrm{~V}$ reveals a starkly different contrast: there now is nearly no signal on the A sub-lattice whereas the $\mathrm{B}$ and $\mathrm{C}$ sites show up more prominently. This is the hallmark of the flat electron band for which the Lieb lattice is known ${ }^{24}$.

We performed tight-binding simulations of the Lieb lattice based on our experimental structure and estimates of the hopping amplitudes. As the next-nearest neighbour coupling (coupling between $\mathrm{B}$ and $\mathrm{C}$ sites) is non-zero, the flat band will be slightly distorted (see Supplementary Information). We estimate the next-nearest neighbour interactions by assuming that the coupling between vacancies depends exponentially on the separation distance to obtain a value of $t_{\mathrm{NNN}} \approx 0.045 \mathrm{eV}$. The lower panel of Fig. 3 shows the results of the tight-binding simulation. A good agreement with the experiment is found using our coupling estimates of $t_{\mathrm{NN}} \approx 0.14 \mathrm{eV}$ and $t_{\mathrm{NNN}} \approx 0.045 \mathrm{eV}$, giving a ratio of $t_{\mathrm{NNN}} / t_{\mathrm{NN}} \approx 0.33$ (see Supplementary Information for details).

In summary, we have presented a general approach for producing tailor-made band structures through atom manipulation using STM. We implemented two model systems with topological states and nearly flat electron bands in a precisely controlled environment. Our approach, combined with the possibilities of automating structure building at the atomic level ${ }^{20}$, places a vast amount of relevant model systems within experimental reach. Additional customizability could come from adapting such assembly techniques to, for example, heavy metals with magnetic properties or significant spin-orbit coupling. The next major challenge is to identify systems in which the energies of the participating states are close to the Fermi level, or can be tuned through the relevant energy range, to observe effects such as the magnetic instability of the flat band or the fractional states of the SSH chain. Further progress in this direction may allow atomic assemblies to produce intriguing physics similar 
a

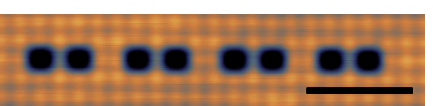

b

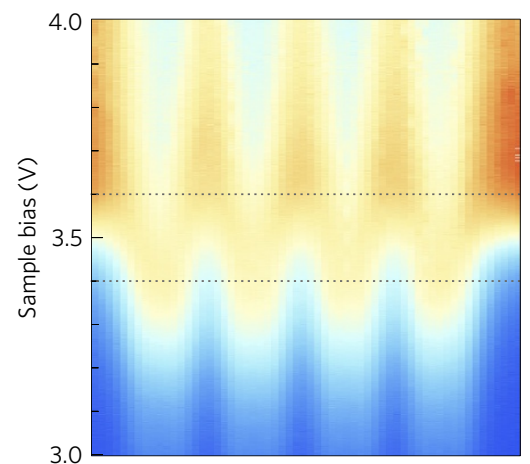

c

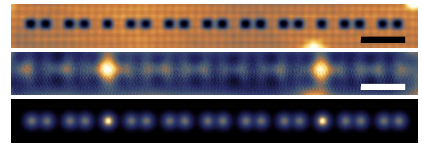

d

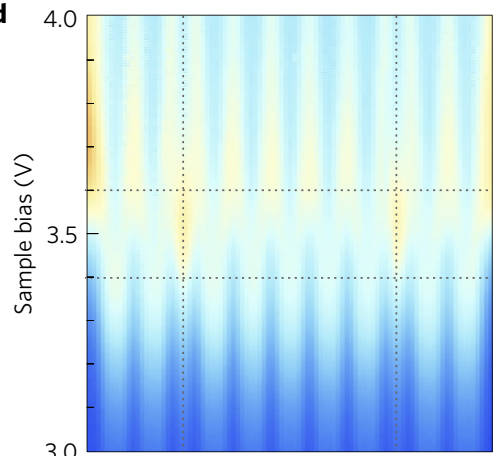

e
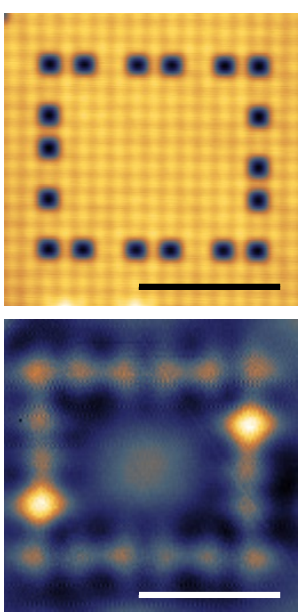

Figure 2 | Domain walls within dimer chains and rings. a, Topography of a dimer chain assembled from vacancy sites. Scale bar, 2 nm. b, Stacked contour plot of point spectra taken along the axis of the chain in $\mathbf{a}$. Red and blue correspond to high and low conductance, respectively. The states split into two bands with no states located within the gap. The dashed lines mark the theoretically expected bandgap. c, Top: topography of a dimer chain with two domain walls separating sections with different topological properties. Middle: constant height conductance map acquired at $3.53 \mathrm{~V}$ (mid-gap) showing two prominent states at the locations of the domain walls. Scale bars, $2 \mathrm{~nm}$. Bottom: simulated LDOS map based on tight-binding calculation. d, Stacked contour plot of point spectra acquired along the axis of the chain in c. Two states appear at the mid-gap energy at the domain-wall locations (vertical dashed lines). The horizontal dashed lines mark the theoretically expected bandgap. e, Top: topography of a ring-like structure with two domain walls. Bottom: conductance map acquired at $3.5 \mathrm{~V}$ showing mid-gap states at the domain-wall locations. Scale bars, $3 \mathrm{~nm}$.

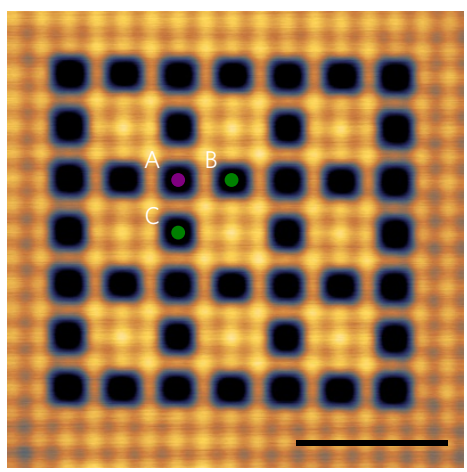

b

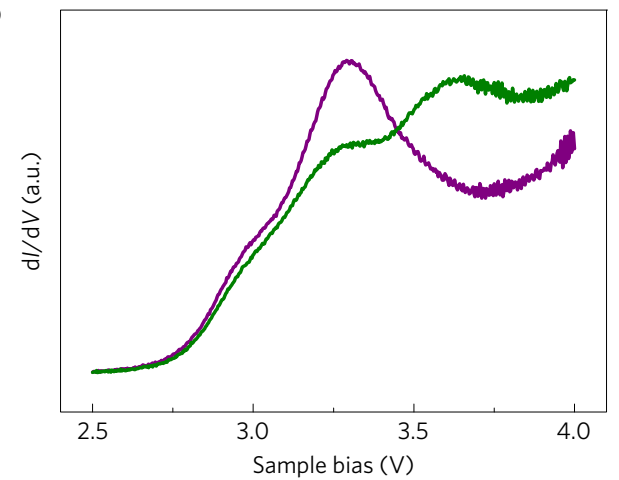

c
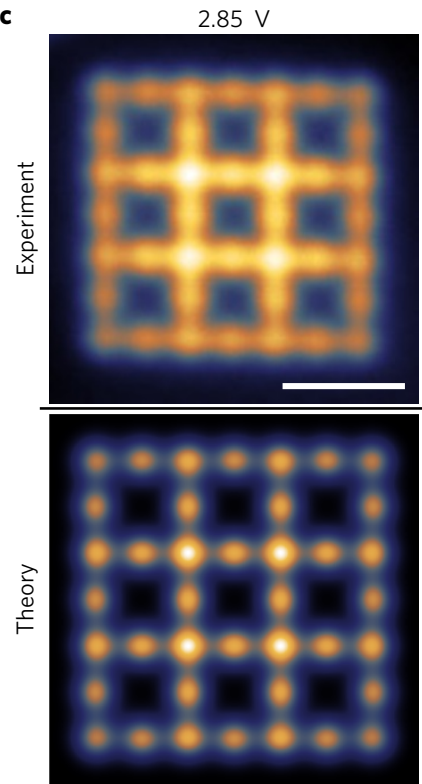

Dispersive band
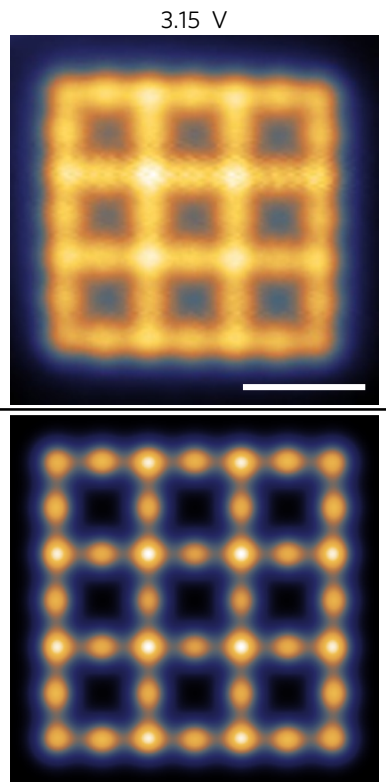

Dispersive band

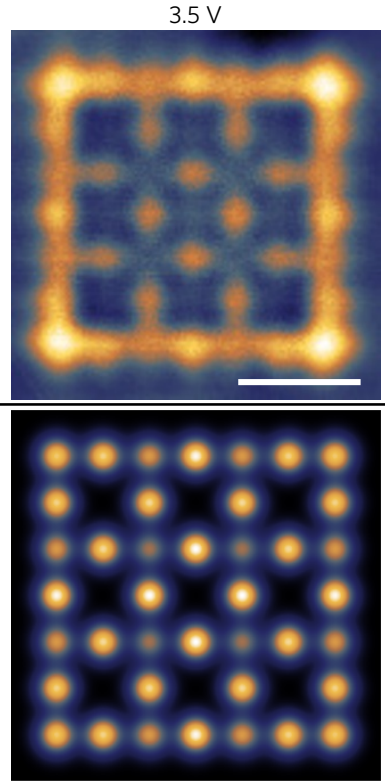

Flat band

Figure $\mathbf{3}$ | Lieb lattice. $\mathbf{a}$, Topography of the Lieb lattice structure assembled from $\mathrm{Cl}$ vacancies. $\mathbf{b}$, Point spectra acquired at the locations indicated in $\mathbf{a}$ c, Conductance maps (top) and tight-binding simulations (bottom) of the Lieb lattice. Bias values (from left to right): $2.85 \mathrm{~V}, 3.15 \mathrm{~V}$ and $3.5 \mathrm{~V}$. The rightmost panel corresponds to the flat band. All scale bars, $2 \mathrm{~nm}$. 
to those seen in ultracold atomic gases while working entirely with electronic states. The same concepts laid out here may be applied to mesoscopic building blocks such as quantum dots to produce quantum materials with tailored properties.

\section{Methods}

Methods, including statements of data availability and any associated accession codes and references, are available in the online version of this paper.

\section{Received 3 November 2016; accepted 24 February 2017;} published online 27 March 2017

\section{References}

1. Kane, C. L. \& Mele, E. J. Quantum spin Hall effect in graphene. Phys. Rev. Lett. 95, 226801 (2005).

2. Bernevig, B. A., Hughes, T. L. \& Zhang, S.-C. Quantum spin Hall effect and topological phase transition in HgTe quantum wells. Science 314, 1757-1761 (2006).

3. Qi, X. L. \& Zhang, S. C. Topological insulators and superconductors. Rev. Mod. Phys. 83, 1-54 (2011)

4. Mourik, V. et al. Signatures of Majorana fermions in hybrid superconductorsemiconductor nanowire devices. Science 336, 1003-1007 (2012).

5. Nadj-Perge, S. et al. Observation of Majorana fermions in ferromagnetic atomic chains on a superconductor. Science 346, 602-607 (2014).

6. Cheon, S., Kim, T.-H., Lee, S.-H. \& Yeom, H. W. Chiral solitons in a coupled double Peierls chain. Science 350, 182-185 (2015).

7. Gomes, K. K., Mar, W., Ko, W., Guinea, F. \& Manoharan, H. C. Designer Dirac fermions and topological phases in molecular graphene. Nature 483, 306-310 (2012).

8. Heeger, A. J., Kivelson, S., Schrieffer, J. R. \& Su, W.-P. Solitons in conducting polymers. Rev. Mod. Phys. 60, 781-851 (1988).

9. Klitzing, K. v., Dorda, Gerhard \& Pepper, M. New method for high-accuracy determination of the fine-structure constant based on quantized Hall resistance. Phys. Rev. Lett. 45, 494-497 (1980).

10. Volovik, G. E. The Universe in a Helium Droplet (Oxford Univ. Press, 2003).

11. König, M. et al. Quantum spin Hall insulator state in HgTe quantum wells. Science 318, 766-770 (2007).

12. Das, A. et al. Zero-bias peaks and splitting in an $\mathrm{Al}-\mathrm{InAs}$ nanowire topological superconductor as a signature of Majorana fermions. Nat. Phys. $\mathbf{8}$, 887-895 (2012)

13. Nadj-Perge, S. et al. Observation of Majorana fermions in ferromagnetic atomic chains on a superconductor. Science 346, 602-607 (2014).

14. Albrecht, S. M. et al. Exponential protection of zero modes in Majorana islands. Nature 531, 206-209 (2016)

15. Schnyder, A. P., Ryu, S., Furusaki, A. \& Ludwig, A. W. W. Classification of topological insulators and superconductors in three spatial dimensions. Phys. Rev. B 78, 195125 (2008).

16. Crommie, M. F., Lutz, C. P. \& Eigler, D. M. Confinement of electrons to quantum corrals on a metal surface. Science 262, 218-220 (1993).

17. Manoharan, H. C., Lutz, C. P. \& Eigler, D. M. Quantum mirages formed by coherent projection of electronic structure. Nature 403, 512-515 (2000).

18. Hirjibehedin, C. F., Lutz, C. P. \& Heinrich, A. J. Spin coupling in engineered atomic structures. Science 312, 1021-1024 (2006).

19. Paavilainen, S., Ropo, M., Nieminen, J., Akola, J. \& Räsänen, E. Coexisting honeycomb and kagome characteristics in the electronic band structure of molecular graphene. Nano Lett. 16, 3519-3523 (2016).
20. Kalff, F. E. et al. A kilobyte rewritable atomic memory. Nat. Nanotech. 11, 926-929 (2016)

21. Repp, J., Meyer, G., Paavilainen, S., Olsson, F. E. \& Persson, M. Scanning tunneling spectroscopy of $\mathrm{Cl}$ vacancies in $\mathrm{NaCl}$ films: strong electron-phonon coupling in double-barrier tunneling junctions. Phys. Rev. Lett. 95, 225503 (2005)

22. Schuler, B. et al. Effect of electron-phonon interaction on the formation of one-dimensional electronic states in coupled Cl vacancies. Phys. Rev. B 91, 235443 (2015).

23. Jackiw, R. \& Rebbi, C. Solitons with fermion number 1/2. Phys. Rev. D 13, 3398-3409 (1976).

24. Niță, M., Ostahie, B. \& Aldea, A. Spectral and transport properties of the two-dimensional Lieb lattice. Phys. Rev. B 87, 125428 (2013).

25. Lieb, E. H. Two theorems on the Hubbard model. Phys. Rev. Lett. 62, 1201-1204 (1989).

26. Weeks, C. \& Franz, M. Topological insulators on the Lieb and perovskite lattices. Phys. Rev. B 82, 085310 (2010).

27. Iglovikov, V. I., Hébert, F., Grémaud, B., Batrouni, G. G. \& Scalettar, R. T. Superconducting transitions in flat-band systems. Phys. Rev. B 90, 094506 (2014)

28. Löthman, T. \& Black-Schaffer, A. M. Universal phase diagrams with superconducting domes for electronic flat bands. Preprint at http://arxiv.org/abs/1611.04893 (2016).

29. Kopnin, N. B., Heikkilä, T. T. \& Volovik, G. E. High-temperature surface superconductivity in topological flat-band systems. Phys. Rev. B 83, 220503 (2011)

30. Julku, A., Peotta, S., Vanhala, T. I., Kim, D.-H. \& Törmä, P. Geometric origin of superfluidity in the Lieb-lattice flat band. Phys. Rev. Lett. 117, 045303 (2016)

31. Goldman, N., Urban, D. F. \& Bercioux, D. Topological phases for fermionic cold atoms on the Lieb lattice. Phys. Rev. A 83, 063601 (2011).

32. Mukherjee, S. et al. Observation of a localized flat-band state in a photonic Lieb lattice. Phys. Rev. Lett. 114, 245504 (2015).

33. Taie, S. et al. Coherent driving and freezing of bosonic matter wave in an optical Lieb lattice. Sci. Adv. 1, e1500854 (2015).

\section{Acknowledgements}

This research made use of the Aalto Nanomicroscopy Center (Aalto NMC) facilities and was supported by the European Research Council (ERC-2011-StG No. 278698 PRECISE-NANO), the Academy of Finland through its Centres of Excellence Program (projects no. 284594 and 284621) and Academy Research Fellow program (no. 256818), and the Aalto University Centre of Quantum Engineering.

\section{Author contributions}

All authors jointly conceived and planned the experiment. R.D. performed the measurements. R.D. and P.L. analysed the STM data. T.O. proposed the SSH dimer chain structure and provided the description of its physics. A.H. proposed the Lieb lattice model and performed the tight-binding calculations for this structure. All authors jointly authored, commented, and corrected the manuscript.

\section{Additional information}

Supplementary information is available in the online version of the paper. Reprints and permissions information is available online at www.nature.com/reprints. Publisher's note: Springer Nature remains neutral with regard to jurisdictional claims in published maps and institutional affiliations. Correspondence and requests for materials should be addressed to P.L.

\section{Competing financial interests}

The authors declare no competing financial interests. 


\section{Methods}

All sample preparations and experiments were carried out in an ultrahigh vacuum system with a base pressure of $\sim 10^{-10}$ mbar. The (100)-terminated copper single crystal was cleaned by repeated cycles of $\mathrm{Ne}^{+}$sputtering at $1.5 \mathrm{kV}$, annealing to $600^{\circ} \mathrm{C}$. To prepare the chloride structure, anhydrous $\mathrm{CuCl}_{2}$ was deposited from an effusion cell held at $300^{\circ} \mathrm{C}$ onto the warm crystal $\left(T \approx 150-200^{\circ} \mathrm{C}\right)$ for $180 \mathrm{~s}$. The sample was held at the same temperature for $10 \mathrm{~min}$ following the deposition.

After the preparation, the sample was inserted into the low-temperature STM (Unisoku USM-1300) and all subsequent experiments were performed at $T=4.2 \mathrm{~K}$. STM images were taken in the constant current mode. $\mathrm{d} I / \mathrm{d} V$ spectra were recorded by standard lock-in detection while sweeping the sample bias in an open feedback loop configuration, with a peak-to-peak bias modulation of $20 \mathrm{mV}$ at a frequency of $709 \mathrm{~Hz}$. Line spectra were acquired in constant height; the feedback loop was not closed at any point between the acquisition of the first and last spectra. Manipulation of the chlorine vacancies was carried out using a procedure adapted from ref. 20. The tip was placed above a $\mathrm{Cl}$ atom adjacent to a vacancy site at $0.5 \mathrm{~V}$ bias voltage and the current was increased to 1 to $2 \mu \mathrm{A}$ with the feedback circuit engaged. The tip was then dragged towards the vacancy site at a speed of up to $250 \mathrm{pm} \mathrm{s}^{-1}$ until a sharp jump in the $z$-position of the tip was observed. This procedure leads to the $\mathrm{Cl}$ atom and the vacancy site exchanging positions with high fidelity.

Data availability. The data that support the plots within this paper and other findings of this study are available from the corresponding author upon reasonable request. 